\title{
Exact fermion zero-mode for the new calorons
}

\author{
M.N. Chernodub ${ }^{\mathrm{a}}$, Thomas C. Kraan ${ }^{\mathrm{b}}$ and Pierre van Baal ${ }^{\mathrm{b} *}$ \\ anstitute of Theoretical and Experimental Physics, \\ B.Cheremushkinskaya 25, Moscow, 117259, Russia \\ bInstituut-Lorentz for Theoretical Physics, University of Leiden, \\ PO Box 9506, NL-2300 RA Leiden, The Netherlands.
}

We construct the fermion zero-mode for arbitrary charge one $S U(n)$ calorons with non-trivial holonomy, both in the finite temperature context (anti-periodic boundary conditions in time) and in the Kaluza-Klein compactification context (periodic boundary conditions in time). The zero-mode is localised on one of the constituent monopoles and we discuss a relation to the Callias index theorem.

\section{Introduction}

The $S U(n)$ instantons at finite temperature (or calorons) can be seen as bound states of $n$ constituent monopoles, evident only when the Polyakov loop at spatial infinity is non-trivial. In the periodic gauge, $A_{\mu}(t+\beta, \vec{x})=A_{\mu}(t, \vec{x})$,

$\mathcal{P}_{\infty}=\lim _{|\vec{x}| \rightarrow \infty} P \exp \left(\int_{0}^{\beta} A_{0}(\vec{x}, t) d t\right)$.

After a suitable constant gauge transformation, it can be characterised by $\sum_{m=1}^{n} \mu_{m}=0$ and

$$
\begin{aligned}
& \mathcal{P}_{\infty}^{0}=\exp \left[2 \pi i \operatorname{diag}\left(\mu_{1}, \ldots, \mu_{n}\right)\right], \\
& \mu_{1} \leq \ldots \leq \mu_{n} \leq \mu_{n+1} \equiv 1+\mu_{1} .
\end{aligned}
$$

Using the classical scale invariance we can always arrange $\beta=1$, as will be assumed throughout. A remarkably simple formula for the $S U(n)$ action density exists [1, 2].

$$
\begin{aligned}
& \operatorname{Tr} F_{\mu \nu}^{2}(x)=\partial_{\mu}^{2} \partial_{\nu}^{2} \log \psi(x), \\
& \psi(x)=\frac{1}{2} \operatorname{tr}\left(\mathcal{A}_{n} \cdots \mathcal{A}_{1}\right)-\cos (2 \pi t), \\
& \mathcal{A}_{m} \equiv \frac{1}{r_{m}}\left(\begin{array}{cc}
r_{m} & \left|\vec{y}_{m}-\vec{y}_{m+1}\right| \\
0 & r_{m+1}
\end{array}\right)\left(\begin{array}{cc}
c_{m} & s_{m} \\
s_{m} & c_{m}
\end{array}\right),
\end{aligned}
$$

with $r_{m}=\left|\vec{x}-\vec{y}_{m}\right|$ the center of mass radius of the $m^{\text {th }}$ constituent monopole, which can be assigned a mass $8 \pi^{2} \nu_{m}$, where $\nu_{m} \equiv \mu_{m+1}-\mu_{m}$. Furthermore, $c_{m} \equiv \cosh \left(2 \pi \nu_{m} r_{m}\right), s_{m} \equiv \sinh \left(2 \pi \nu_{m} r_{m}\right)$, $r_{n+1} \equiv r_{1}$ and $\vec{y}_{n+1} \equiv \vec{y}_{1}$.

\footnotetext{
* Presented by the last author at Lattice '99, Pisa, Italy.
}

\section{Monopole constituents}

These generalised caloron solutions can be found 33 using a combination of the Nahm transformation [4] and the Atiyah-Drinfeld-HitchinManin (ADHM) construction [5]. The latter is mainly needed to resolve the delta function singularities that arise in the Nahm transformation, although other methods were developed as well [6].

The Nahm equation for these charge one instantons reduces to an abelian problem on the circle, parametrised by $z \bmod 1$,

$\frac{d}{d z} \hat{A}_{j}(z)=2 \pi i \sum_{m}\left(y_{m}^{j}-y_{m-1}^{j}\right) \delta\left(z-\mu_{m}\right)$,

giving $\hat{A}_{j}(z)=2 \pi i y_{m}^{j}$, for $z \in\left[\mu_{m}, \mu_{m+1}\right]$. In the monopole literature $\hat{A}_{j}(z)$ is usually denoted by $T_{j}(z)$. Taking one interval in isolation, applying the Nahm transformation [4] gives a single static Bogomol'nyi-Prasad-Sommerfeld (BPS) monopole with mass proportional to the length $\left(\nu_{m}\right)$ of the interval. Taking $\left|\vec{y}_{n}\right| \rightarrow \infty$ leaves the interval $\left[\mu_{1}, \mu_{n}\right]$, allowing for the interpretation of an $S U(n)$ monopole with $\mu_{i}$ specifying the eigenvalues of the Higgs field at infinity, for which it is crucial they add to zero. Indeed, in the periodic gauge $A_{0}$ tends to a constant at spatial infinity.

Note that we have to order $\exp \left(2 \pi i \mu_{m}\right)$ along the circle to ensure that the $\nu_{i}$ add to 1 , an ordering inherited by $\mu_{m}$ when extended to the real line by insisting $\mu_{k n+m}=k+\mu_{m}$, for any 
integer $k$. Let us pick one to be labelled by $\mu_{1}$. All we can guarantee at this point is that $\sum_{m=1}^{n} \mu_{m}=\ell$, an integer. With $\mu_{k n+m}=k+\mu_{m}$, we find $\sum_{m=1}^{n} \mu_{m+p}=\ell+p$, for any integer $p$. A cyclic shift of the labels by $p=-\ell$ proves that there is a unique choice of the $\mu_{m}$ that satisfy eq. (2). It demonstrates why $\vec{y}_{n}$ does play a special role, and in the limit $\left|\vec{y}_{n}\right| \rightarrow \infty$ one therefore has a static monopole solution 低, which can be seen as the composite of $n-1$ BPS monopoles of mass $\nu_{m}$, located at $\vec{y}_{m}$, for $m=1, \cdots, n-1$. From the general formalism it is clear these $n-1$ monopole constituents are time independent, as was verified explicitly for $S U(2)$ [2, 3]. Note that our argument demonstrates that for $\left|\vec{y}_{m}\right| \rightarrow \infty$ with $m \neq n$, one is left with a gauge field that cannot be time independent, even though the resulting action density is [2].

The significance of one constituent carrying a time dependent field lies in the fact that the $n$ constituent monopoles form an instanton, and the topological charge can be associated to the socalled Taubes-winding [7], described by a time dependent (gauge) rotation, going full circle when $t$ progresses over one period. For $S U(2)$ this can be read-off from the explicit expression for the gauge field [2.,3]. We thus conclude that the constituent located at $\vec{y}_{n}$ is the one that carries this Taubes-winding, even though its action density is time independent for well-separated constituents. This conclusion can also be drawn from the formalism developed in ref. [6], see also ref. [8].

\section{Fermion zero-mode}

The basic ingredient in the construction of caloron solutions is a Greens function defined by

$$
\left(D_{z}^{2}+r^{2}(x ; z)+\sum_{m} \delta_{m}(z)\right) \hat{f}_{x}\left(z, z^{\prime}\right)=\delta\left(z-z^{\prime}\right),
$$

where $D_{z}=(2 \pi i)^{-1} \partial_{z}-t, r^{2}(x ; z)=r_{m}^{2}(x)$ for $z \in$ $\left[\mu_{m}, \mu_{m+1}\right]$ and $\delta_{m}(z)=\delta\left(z-\mu_{m}\right)\left|\vec{y}_{m}-\vec{y}_{m-1}\right| / 2 \pi$. A similarity with the impurity scattering problem allows for a straightforward solution [1], which we present here for the case that $\mu_{m} \leq z^{\prime} \leq z \leq \mu_{m+1}$ (extended to $z<z^{\prime}$ by $\hat{f}_{x}\left(z^{\prime}, z\right)=\hat{f}_{x}^{*}\left(z, z^{\prime}\right)$ )

$$
\hat{f}_{z}\left(z, z^{\prime}\right)=\frac{\pi e^{2 \pi i t\left(z-z^{\prime}\right)}}{r_{m} \psi}\left(e^{-2 \pi i t} \sinh \left(2 \pi\left(z-z^{\prime}\right) r_{m}\right)+\right.
$$

$$
\left.<v_{m}\left(z^{\prime}\right)\left|\mathcal{A}_{m-1} \cdots \mathcal{A}_{1} \mathcal{A}_{n} \cdots \mathcal{A}_{m}\right| w_{m}(z)>\right),
$$

where the spinors $v_{m}$ and $w_{m}$ are defined by

$$
\begin{aligned}
& v_{m}^{1}(z)=-w_{m}^{2}(z)=\sinh \left(2 \pi\left(z-\mu_{m}\right) r_{m}\right), \\
& v_{m}^{2}(z)=w_{m}^{1}(z)=\cosh \left(2 \pi\left(z-\mu_{m}\right) r_{m}\right) .
\end{aligned}
$$

For the zero-mode densities we find

$\left|\Psi_{z}(x)\right|^{2}=-(2 \pi)^{-2} \partial_{\mu}^{2} \hat{f}_{x}(z, z)$,

derived exactly as for $S U(2)$ [9], not repeated here. With the gauge field in the periodic gauge one has, $\Psi_{z}(t+1, \vec{x})=\exp (2 \pi i z) \Psi_{z}(t, \vec{x})$. To obtain the finite temperature fermion zero-mode one puts $z=\frac{1}{2}$, whereas for the fermion zero-mode with periodic boundary conditions, relevant in supersymmetric applications, one takes $z=0$.

In figure 1 we show a typical $S U(3)$ caloron, illustrating that also for $n>2$ the fermion zeromodes are localised on one of the constituents. This localisation can be established easily in the

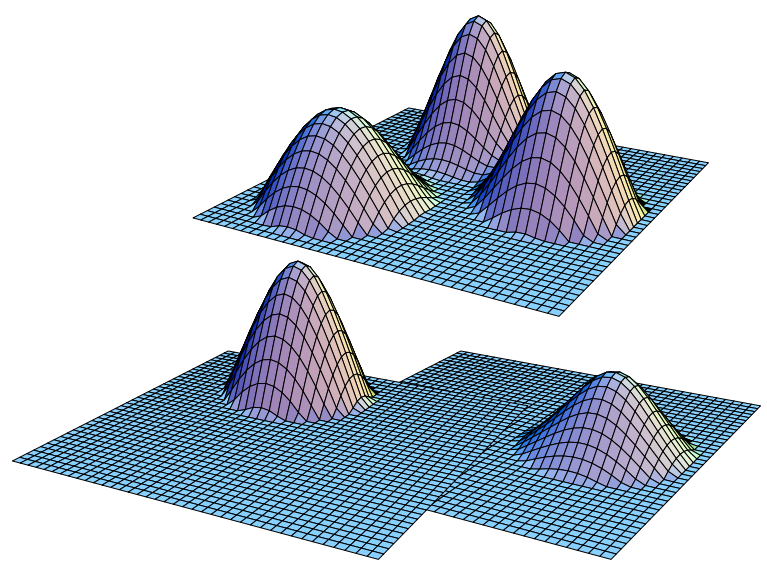

Figure 1. The action densities (top) for the $S U(3)$ caloron, cut off at $1 /(2 e)$, on a logarithmic scale, with $\left(\mu_{1}, \mu_{2}, \mu_{3}\right)=(-17,-2,19) / 60$ for $t=0$ in the plane defined by $\vec{y}_{1}=(-2,-2,0)$, $\vec{y}_{2}=(0,2,0)$ and $\vec{y}_{3}=(2,-1,0)$, for $\beta=1$, with masses $8 \pi^{2} \nu_{i},\left(\nu_{1}, \nu_{2}, \nu_{3}\right)=(0.25,0.35,0.4)$. On the bottom-left is shown the zero-mode density for fermions with anti-periodic boundary conditions in time and on the bottom-right for periodic boundary conditions, at equal logarithmic scales, cut off below $1 / e^{5}$. 
limit of large $\left|\vec{y}_{i}-\vec{y}_{i+1}\right|$ for all $i$, in which case one finds, when $z \in\left[\mu_{m}, \mu_{m+1}\right]$,

$\hat{f}_{x}(z, z)=\frac{\sinh \left[2 \pi\left(z-\mu_{m}\right) r_{m}\right] \sinh \left[2 \pi\left(\mu_{m+1}-z\right) r_{m}\right]}{r_{m} \sinh \left[2 \pi \nu_{m} r_{m}\right] / 2 \pi}$

making explicit that the location of the zero-mode is determined by the interval that contains the appropriate value of $z$. From eq. (2) it follows that $\mu_{1} \leq 0 \leq \mu_{n}$, such that the periodic zero-mode is associated to the static constituent at $\vec{y}_{m}$, with $\mu_{m} \leq 0 \leq \mu_{m+1}$. This is precisely the condition for the existence of a zero-mode given by the Callias index theorem [10] (see also the appendix of ref. [11]). Due to the static background (for wellseparated constituents), time dependence of the zero-mode would be of the form $\exp (2 \pi i k t)$ for $k$ integer, shifting $z=0$ by $k$, out of the interval that allows for a zero-mode.

Allowing for $k= \pm \frac{1}{2}$, for which $\exp (2 \pi i k t)$ turns the periodic zero-mode anti-periodic, we can have situations where this anti-periodic zero-mode is associated to one of the static monopole constituents. A specific example for $S U(3)$ where this occurs is $\left(\mu_{1}, \mu_{2}, \mu_{3}\right)=(-0.48,-0.03,0.51)$, yielding $\left(\nu_{1}, \nu_{2}, \nu_{3}\right)=(0.45,0.54,0.01)$. Both the periodic and the anti-periodic zero-mode are associated to the $2^{\text {nd }}$ constituent. We note that, apart from the fact that the $3^{\text {rd }}$ constituent is nearly massless, both zero-modes are very broad since $\min \left(z-\mu_{2}, \mu_{3}-z\right)=0.03$ for $z=0$ and 0.01 for $z=\frac{1}{2}$. For $S U(2) z=0$ is always midway between $\mu_{1}$ and $\mu_{2}$ and $z=\frac{1}{2}$ midway between $\mu_{2}$ and $\left.\mu_{3}=1+\mu_{1}\right)$. When $z$ coincides with $\mu_{i}$, the zero-mode is no longer normalisable, which is the origin of the delta function singularities in the Nahm transformation.

\section{Conclusions}

In conclusion, for well-separated constituents the fermion zero-mode is localised to a single constituent. For $S U(2)$ the anti-periodic zero-mode is always associated to the constituent that carries Taubes-winding [9]. For $S U(n>2)$ this is also typically true (see fig. 1), in particular when well localised, something that may be significant for developing a model for the QCD vacuum that combines monopoles and instantons 2, 3, :9. How- ever, exceptions exist where both the periodic and anti-periodic zero-mode are associated to (possibly the same) static constituent(s), although this tends to be accompanied by nearly massless constituents, and rather delocalised zero-modes.

\section{Acknowledgements}

We thank, Jan de Boer for pointing us to the connection with the Callias theorem, Tamás Kovács and Mikhail Voloshin for discussions, Diego Bellisai for correspondence and Margarita García Pérez for collaboration in the early stages. MNCh thanks the Theoretical Physics Institute at the University of Minnesota and the InstituteLorentz for hospitality. This work was supported in part by INTAS grant RFBR 95-0681. MNCh was supported by the INTAS fellowship YSF 9895 and TCK by a grant from FOM/SWON. PvB thanks the Aspen Center of Physics, where part of this work was done, and workshop organisers Urs Heller and Rajamani Narayanan, and participants, for creating a stimulating atmosphere.

\section{REFERENCES}

1. T.C. Kraan and P. van Baal, Phys. Lett. B435 (1998) 389.

2. T.C. Kraan and P. van Baal, Nucl. Phys. B(Proc.Suppl.) 73 (1999) 554.

3. T.C. Kraan and P. van Baal, Phys. Lett. B428 (1998) 268; Nucl. Phys. B533 (1998) 627.

4. W. Nahm, Self-dual monopoles and calorons, in: Lecture Notes in Physics 201 (1984) 189.

5. M.F. Atiyah, N.J. Hitchin, V. Drinfeld and Yu.I. Manin, Phys. Lett. 65A (1978) 185

6. K.Lee and P. Yi, Phys. Rev. D56(1997)3711; K.Lee and C.Lu, Phys.Rev.D58(1998)025011.

7. C. Taubes, in: Progress in gauge field theory, eds. G.'t Hooft e.a., (Plenum Press, New York, 1984) p.563

8. N.M. Davies, T.J. Hollowood, V.V. Khoze and M.P. Mattis, hep-th/9905015.

9. M.García Pérez, A.González-Arroyo, C.Pena and P. van Baal, Phys.Rev. D60(1999)031901.

10. C.J. Callias, Comm.Math.Phys. 62(1978)213.

11. J. de Boer, K. Hori and Y. Oz, Nucl. Phys. B500 (1997) 163. 